\title{
Cepstral Characteristics of Voice before, during and after Menopause: A Cross-sectional Study
}

\author{
${ }^{1}$ Radish Kumar Balasubramanium, ${ }^{2}$ Jayashree $S$ Bhat
}

\begin{abstract}
Background: Previous studies on effects of menopause on voice characteristics have not compared the voice characteristics before, during and after menopause. It is also possible that these voice changes cannot be captured by traditional acoustic measures such as frequency and amplitude perturbation measures. Hence a robust acoustic tool such as cepstrum was chosen to capture these vocal changes before, during and after menopause and hence the present study was attempted.
\end{abstract}

Method: The present study followed a cross-sectional study design. The participants consisted of 25 premenopausal women, 25 perimenopausal women and 25 postmenopausal women. Sustained production of/a/ and narration was recorded using $\mathrm{Z}$ tool and cepstral peak prominence (CPP) and smoothened cepstral peak prominence (CPPS) was analyzed.

Results: The results of one-way ANOVA revealed that there was a significant main effect of the group at $p<0.05$ for all the cepstral measures targeted in the study. Post hoc analysis indicated that there was no significant difference between pre and perimenopausal women for all the cepstral measures at $p<0.05$. However, there was a significant difference between pre and postmenopausal women and peri and postmenopausal women at $p<0.05$. These results suggests that postmenopausal women had overall good voice quality than pre and perimenopausal women.

Conclusion: Results indicated that post menopausal women had increased cepstral measures when compared to pre and perimenopausal women. Further, studies are required to correlate laryngeal examination and hormonal levels with that of the voice changes before, during and after menopausal women.

Keywords: Menopause, Voice, Analysis, Cross-sectional study. How to cite this article: Balasubramanium RK, Bhat JS. Cepstral Characteristics of Voice before, during and after Menopause: A Cross-sectional Study. Int J Phonosurg Laryngol 2014;4(2):50-54.

Source of support: Nil

Conflict of interest: None

\footnotetext{
${ }^{1}$ Associate Professor, ${ }^{2}$ Professor

1,2Department of Audiology and Speech Language Pathology Kasturba Medical College, Manipal University, Mangalore Karnataka, India

Corresponding Author: Radish Kumar Balasubramanium Associate Professor, Department of Audiology and Speech Language Pathology, Kasturba Medical College, Manipal University, Mangalore, Karnataka, India, Phone: 08244286432 e-mail: radheesh_b@yahoo.co.in
}

\section{INTRODUCTION}

The vocal folds have often been reported as sensitive to hormonal changes. ${ }^{1-3}$ The physiologic effects of the menstrual cycle on voice have been researched and it was found to influence voice. ${ }^{1,2,4,5}$ Similarly, it is also possible that the hormones regulating the menopause could also create changes in the voice before, during, or after menopause leading to dysphonia in these population. However, the effects of menopause on voice characteristics are debatable due to the ambiguous findings available in the literature.

Boulet and Oddens reported voice changes such as huskiness and difficulty in reaching higher registers around the time of menopause in 48 females in the age range of 40 to 70 years through questionnaire survey. However, these changes in voice were not subjected to detailed acoustic and perceptual analysis. ${ }^{6}$ Schneider et $\mathrm{al}^{7}$ reported that 49 out of 107 women reported voice changes postmenopause. Voice changes reported by these 49 individuals include throat dryness, frequent throat clearing, lower frequency levels and increased hoarseness. These changes were further substantiated with the abnormal findings in perceptual voice evaluation, videostroboscopy, voice range profile and voice dysfunction index. In contrast, Meurer et $\mathrm{al}^{8}$ reported no significant variations in fundamental frequency and vocal intensity in postmenopausal women. Sovani and Mukundhan, ${ }^{9}$ investigated the voice characteristics in postmenopausal women in professional voice users. Results revealed that there was decline in fundamental frequency, standard fundamental frequency and maximum phonation time while noise to harmonic ratio and voice turbulence index increased in postmenopausal women. It was also concluded that the presence and absence of voice use in ones profession had differential effect on the post menopausal voice changes. ${ }^{9}$ However, these studies have not compared the premenopausal and postmenopausal women for voice changes.

D'haeseeler et $\mathrm{al}^{10}$ compared the voice characteristics in premenopausal and postmenopausal women and the results indicated that the postmenopausal women had overall good voice quality in comparison to premenopausal women except that they had lower fundamental frequency. D'haeseeler et al ${ }^{11}$ studied the voice character- 
istics in middle aged premenopausal women during the menopause transition and the results revealed that the premenopausal women displayed a reduced frequency and intensity range, decreased habitual fundamental frequency, and an increased soft phonation index in comparison to the young women. They also concluded that these results are important in the differential diagnosis of vocal changes caused by aging and menopause.

Hormonal therapy is a commonly used therapy for women with menopausal complaints. However, it was not clear whether hormonal therapy could improve the menopausal voice complaints. In this regard, Mendes-Laureano et $\mathrm{al}^{12}$ investigated the effect of menopause on voice in menopausal women with and without hormonal therapy on fundamental frequency parameter. Results showed no significant differences in fundamental frequency with respect to menopause and the use of hormal therapy. In the similar line, D'haeseleer et $\mathrm{al}^{13}$ investigated the impact of HT on vocal characteristics in postmenopausal women by comparing postmenopausal women with and without hormonal therapy. Postmenopausal women without hormonal therapy showed a significantly lower speaking fundamental frequency compared to postmenopausal women with hormonal therapy with the difference of around $14 \mathrm{~Hz}$. This indicated that hormonal therapy can neutralize voice changes caused due to menopause. It was also observed that there were no significant differences observed between estrogen therapy and estrogen and progestogen therapy. However, the sample size considered in both groups of hormonal therapy were heterogeneous. Similar study was undertaken by the same group of authors by keeping body mass index into consideration and the results indicated a drop in the speaking fundamental frequency only in post menopausal women without undertaking any hormonal therapy and with a low body mass index. Authors concluded that the factors like adipose tissue (High BMI) and hormonal therapy (different types of hormonal therapy, different concentrations of hormonal therapy) would counteract the drop in speaking fundamental frequency in postmenopausal women. ${ }^{14,15}$

None of the above-mentioned studies have compared the voice characteristics before, during and after menopause. Also, it is not known to what extent these changes will influence the voice quality. It is possible that these changes cannot be captured by traditional acoustic measures such as frequency and amplitude perturbation measures. Hence, a robust acoustic tool such as cepstrum is needed to capture these vocal changes before, during and after menopause. Cepstrum is a result of fast fourier transformation of a spectrum. As cepstrum does not rely on the measurement of fundamental frequency, it tends to be more reliable than the traditional perturbation measures (Hillenbrand, 1987). These measures give a better picture of degree of harmonic organization and better predict dysphonia than the traditional jitter and shimmer measures. ${ }^{16-19}$ In view of majority of the past studies have focussed on the voice characteristics only in post menopausal women, and hence the present study attempts to analyze the cepstral characteristics of voice before, during and after menopause in women using a cross-sectional study design. Cross-sectional study design was chosen as it saves time, minimizes the expenses and avoid attrition during longitudinal follow-ups.

\section{METHOD}

The present study followed a cross-sectional study design.

\section{Participants}

The participants were divided into three groups. Group 1 consisted of 25 premenopausal women in the age range of 38 to 42 years (Mean age $=39.8 ; \mathrm{SD}=1.41$ ). All these participants were having regular periods in their menstrual cycle. Group 2 consisted of 25 perimenopausal women in the age range of 40 to 50 years (mean age $=$ $45.52 ; \mathrm{SD}=2.86$ ). These participants were having irregular periods indicating that they were on their way toward the attainment of menopause. Group 3 consisted of 25 postmenopausal women in the age range of 49 to 52 years (mean age $=50.48 ; \mathrm{SD}=1.15$ ). All the individuals in group 3 participants had 1 to 3 years postmenopause.

None of these participants had the history of vocal abuse/misuse, hormonal problems, respiratory illness, exposure to toxic fumes, smoking and alcohol consumption as ascertained through detailed case history. All these participants had normal weight as measured through body mass index and were not undertaking any hormonal therapy for menopause. All these participants had normal voice quality with respect to their age and gender through GRBAS rating scale ${ }^{20}$ by three experiences speech language pathologists at our hospital who are unaware of the purpose of the study. The intrajudge and interjudge reliability of these ratings were found to be 95 and $96 \%$ respectively. Voice disorder-outcome profile (V$\mathrm{DOP})^{21}$ also indicated no handicap associated with their voices in any of the three groups.

\section{Instrumentation}

$\mathrm{Z}$ tool or speech tool given by Hillenbrand was used to record and analyze cepstral peak prominence (CPP) and smoothened cepstral peak prominence (CPPS) during phonation and narration. 


\section{Procedure}

Participants were seated comfortably on a chair and the sustained phonation of /a/ was recorded. They were also asked to narrate about their home for 1 minute. Microphone was kept $15 \mathrm{~cm}$ away from the participants mouth while recording. Three recordings of sustained phonation of /a/ were recorded. The entire voice sample was analyzed separately for phonation and narration using $\mathrm{z}$ tool and the parameters such as CPP and CPPs for sustained phonation of vowel /a/ and CPPCS, and CPPCSs for narration sample (CS here refers to continuous speech; s refers to smoothened) were extracted using $\mathrm{z}$ tool.

\section{Data Analysis}

The software directly measures the following parameters using the Hillenbrand algorithm.

Cepstral peak prominence (CPP): The CPP is the difference in amplitude between the cepstral peak and the corresponding value on the regression line that is directly below the peak.

Smoothened cepstral peak prominence: CPPS is defined as the average of the individual cepstra over a given number of frames before extracting the cepstral peak and calculating the peak prominence.

Both CPP and the CPPS have been shown to correlate with perceptions of breathiness, with CPPS being the better predictor of vocal impairments. (Yolanda, Deirdre, and George S, 2002). Average value of CPP, CPPs, CPPCS, CPPCSs were taken. The best of the three trials among the sustained vowel production task was considered for statistical analysis.

\section{Statistical Analysis}

Mean and standard deviation for all the cepstral measures were calculated for each participant in all the three groups. One-way ANOVA was employed to find out the significant main effect of the groups followed by Bonferronis post hoc test.

\section{RESULTS}

The voice changes before, after and during menopause was analyzed using descriptive statistics and one-way ANOVA. The results of descriptive statistics were shown in the Table 1.

From the table it is observed that there were not much of differences between pre and perimenopausal women. However, postmenopausal women differed from the other groups in all the cepstral measures.

The results of one-way ANOVA revealed that there was a significant main effect of the group at $p<0.05$ for
Table 1: Mean and standard deviation of cepstral measures in premenopausal, perimenopausal and postmenopausal women

\begin{tabular}{|c|c|c|c|c|c|c|}
\hline \multirow[b]{2}{*}{ Parameters } & \multicolumn{2}{|c|}{$\begin{array}{c}\text { Pre- } \\
\text { menopausal } \\
\text { women }\end{array}$} & \multicolumn{2}{|c|}{$\begin{array}{c}\text { Peri- } \\
\text { menopausal } \\
\text { women }\end{array}$} & \multicolumn{2}{|c|}{$\begin{array}{c}\text { Post } \\
\text { menopausal } \\
\text { women }\end{array}$} \\
\hline & Mean & $S D$ & Mean & $S D$ & Mean & $S D$ \\
\hline CPP & 17.73 & 1.92 & 17.37 & 2.88 & 19.29 & 1.74 \\
\hline CPPS & 3.87 & 0.61 & 3.80 & 1.01 & 4.52 & 0.61 \\
\hline CPPCS & 12.43 & 1.12 & 12.37 & 1.27 & 14.29 & 1.17 \\
\hline CPPCSS & 3.34 & 0.72 & 3.53 & 1.18 & 4.25 & 0.68 \\
\hline
\end{tabular}

CPP: Cepstral peak prominence; CPPS: Smoothed cepstral peak prominence; CPPCS: Cepstral peak performance continuous speech; CPPCSS: Smoothened cepstral peak performance continuous speech; SD: Standard deviation

all the cepstral measures targeted in the study. Post hoc analysis indicated that there was no significant difference between pre and perimenopausal women for all the cepstral measures at $p<0.05$. However, there was a significant difference between pre- and postmenopausal women and peri and postmenopausal women at $\mathrm{p}<0.05$. These results suggest that postmenopsuasl women had more dominant cepstral rahmonics than pre- and perimenopausal women.

\section{DISCUSSION}

Sex hormone fluctuations were shown to affect female vocal folds and laryngeal function. ${ }^{22}$ These laryngeal changes are seen throughout the span of life, starting from puberty with the awakening of the hormonal system, fluctuating systematically during the reproductive years with the menstrual cycle, and then changing again with the decline of the hormonal activity during the menopause. Variations in female vocal production that occur during menstruation, pregnancy and menopause all coincide with marked hormonal changes. ${ }^{23}$ Studies have reported that the hormonal variations during menopause can affect the vocal fold vibratory pattern..$^{6-15}$ Majority of the previous studies have reported the voice characteristics only in postmenopausal women using traditional acoustic measures and hence the present study attempted to analyze the voice before, during and after menopause in women using cepstral measures.

The results revealed that there were no significant difference in cepstral measures between premenopausal and perimenopausal women at $\mathrm{p}>0.05$. In the present study, reduction in cepstral measures were also observed in women during their premenopausal period in the age range of 38 to 42 years. This suggests that voice changes are evident just prior to perimenopausal period. This is possible with the rise in follicle stimulating hormone in the presence of regular periods indicating the early stage of perimenopausal period. However, these women were categorized under premenopausal group in the present 
study. This is difficult to be determined unless hormonal analysis is performed on these women. Also, it is not clear at what age during their premenopausal period these women begin developing voice changes which requires further investigation.

Reduced cepstral measures in perimenopausal group could be because of the hormonal fluctuations occurring during the occurrence of menopause. Amir and BironShental ${ }^{22}$ also stated that sex hormone variations can affect female vocal folds and larynx during menopause. D'haeseeler et al also had observed vocal changes during perimenopausal period as a result of hormonal fluctuations. ${ }^{11}$ These hormonal fluctuations are characterized by increase in the follicle stimulating hormone and irregular menstruation period due to the decline in the number of ovarian follicles indicating the late perimenopausal period. However, this is not supported with the hormonal analysis findings in the present study.

Cepstral measures increases after the attainment of menopause leading to improved voice quality in post menopausal women. This finding is supported by a significant difference in cepstral measures between postmenopausal women and pre and perimenopausal women. Increase in the cepstral measures could be due to the stabilization of hormonal levels postmenopause which would have improved the voice quality, thereby increasing the dominant rahmonics. However, it is to be kept in mind that there is a wide range of variability in the voice characteristics due to aging and/or menopausal status. It is also possible that the smaller sample size in comparison to overall population would be the cause for this findings. Moreover, the differences in cepstral measures for narration sample across the groups may be confounded by the fact that the elicited speech samples differed in linguistic/ phonetic context from subject to subject.

These results are in consonance with the previous study by D'haeseeler et al who reported that the post menopausal women had overall good voice quality in comparison to premenopausal women. D'haeseeler et al, ${ }^{10}$ also reported that there were no organic pathologies in postmenopausal women which could also be one of the reasons for improved voice quality, in postmenopausal women. However, the present study could not perform videostroboscopy on any of these participants due to the financial constraints. The present finding did not support the observations of abnormal voice findings in postmenopausal women which is in contrast to the past studies on postmenopausal women. ${ }^{6,7,9}$ which could partially be attributed to the lack of differentiation between postmenopausal voice changes and age related changes. Most of these studies have taken a wide age range of postmenopausal women which could have demonstrated vocal changes in their studies. Even in the present study, it is difficult to differentiate menopause and age related changes in the study participants. ${ }^{24,25}$

The present study carries two limitations which include lack of laryngeal examination and analysis of hormones. Both of which were not contemplated due to the lack of financial support for carrying out the study. Also, the hormone analysis facility was not available at our institution. Despite these problems, the present study highlights the importance of cepstral analysis in the identification of vocal problems before, during and after menopause. This finding also cues the need for voice care during pre and perimenopausal period to preserve the laryngeal apparatus, especially in professional voice users where subtle changes in voice could affect their voice performance in concerts.

\section{CONCLUSION}

The present study examined the cepstral characteristics of voice before, during and after menopause using a crosssectional study paradigm. Results indicated that post menopausal women had increased cepstral measures when compared to pre and perimenopausal women. This could be attributed to the reduction in the hormonal fluctuations after the attainment of menopause. Further, studies are required to correlate laryngeal examination and hormonal levels with that of the voice changes before, during and after menopausal women using a longitudinal study paradigm.

\section{ACKNOWLEDGMENT}

The authors would like to thank the Dean, Kasturba Medical College, Manipal University, Mangalore, for permitting them to carry out the study.

\section{REFERENCES}

1. Abitbol J, Brux J, Millot G, et al. Does a hormonal vocal cord cycle exist in women? Study of vocal premenstrual syndrome in voice performers by videostroboscopy-glottography and cytology on 38 women. J Voice 1989;3(2):157-162.

2. Chae SW, Choi G, Kang HJ, Choi JO, Jin SM. Clinical analysis of voice change as a parameter of premenstrual syndrome. J Voice 2001;15(2):278-283.

3. Altman K, Haines K, Vakkalanka S, et al. Identification of thyroid hormone receptors in the human larynx. Laryngoscope 2003;113(11):1931-1934.

4. Figueiredo LCG, Gonc,alves MRI, Pontes A, Pontes P. Vocal behavior during menstrual cycle: perceptual-auditory, acoustic and self-perception analysis. Rev Bras Otorrinolaringol 2004;70(3):331-339.

5. Abitbol J, Abitbol P, Abitbol B. Sex hormones and the female voice. J Voice 1999;13(3):424-446.

6. Boulet MJ, Oddens BJ. Female voice changes around and after menopause - an initial investigation. Mauritas 1996;23(1):15-21. 
7. SchneiderB, vanTrotsenburgM,HankeG,BigenzahnW,HuberJ. Voice impairment and menopause. Menopause 2004;11(2): 151-158.

8. Meurer EM, Wender MCO, Corleta HE, Capp E. Phonoarticulatory variations of women in reproductive age and postmenopausal. J Voice 2003;18(3):369-374.

9. Sovani P, Mukundhan G. Comparison of postmenopausal voice changes across the professional and nonprofessional users of the voice. South African J Communication Disorders 2010;57(1):76-81.

10. D'haeseleer E, Depypere H, Claeys S. The impact of the menopause on vocal quality. Menopause 2011a;18(3):267-272.

11. D'haeseleer E, Depypere H, Claeys S. Vocal characteristics of middle-aged premenopausal women. J Voice 2011b;25(3): 360-366.

12. Mendes-Laureano J, Sá MF, Ferriani RA, Reis RM, AguiarRicz LN, Valera FC, Küpper DS, Romão GS. Comparison of fundamental voice frequency between menopausal women and women at menacme. Maturitas 2006;55(2):195-199.

13. D'haeseleer E, Depypere H, Claeys S, Baudonck N, Van Lierde K. The impact of hormone therapy on vocal quality in postmenopausal women. J Voice 2012;26(5):671-677.

14. D'haeseleer E, Depypere H, Claeys S, Van Lierde K. The relation between body mass index and speaking fundamental frequency in premenopausal and postmenopausal women. Menopause 2011;18(7):754-758.

15. D'haeseleer E, Depypere H, Van Lierde K. Comparison of speaking fundamental frequency between premenopausal women and postmenopausal women with and without hormone therapy. Folia Phoniatr Logop 2013;65(2):78-83.
16. Heman-Ackah YD, Heuer RJ, Michael DD, et al. Cepstral peak prominence: a more reliable measure of dysphonia. Ann Otol Rhinol Laryngol 2003;112(4):324-333.

17. Heman-Ackah YD, Michael DD, Goding GS Jr. The relationship between cepstral peak prominence and selected parameters of dysphonia. J Voice 2002;16(1):20-27.

18. Heman-Ackah YD. Reliability of calculating the cepstral peak without linear regression analysis. J Voice 2004;18(2):203-208.

19. Zieger K, Schneider C, GerullG,MrowinskiD. Cepstrum analysis in voice disorders. Folia Phoniatr Logop 1994;47(4):210-217.

20. Hirano M. Psychoacoustic evaluation of voice: GRBAS Scale for evaluating the hoarse voice. Clinical examination of voice, Springer Verlag. 1981.

21. Konnai RM, Jayaram M, Scherer RC. Development and validation of a voice disorder outcome profile for an Indian population. J Voice 2010;24(2):206-220.

22. Amir O, Biron-Shental T. The impact of hormonal fluctuations on female vocal folds. Current opinion in otolaryngology and head and neck surgery 2004;12(3):180-184.

23. Caruso S, Roccasalva L, Sapienza G, Zappala M, Nuciforo G, Biondi S. Laryngeal cytological aspects in women with surgically induced menopause who were treated with transdermal estrogen replacement therapy. Fertility and Sterility 2000;74(6):1073-1079.

24. Sataloff RT, Rosen DC, Hawkshaw M, Spiegel JR. The aging adult voice. J Voice 1997;11(2):156-160.

25. da Silva PT, Master S, Andreoni S, Pontes P, Ramos LR. Acoustic and long-term average spectrum measures to detect vocal aging in women. J Voice 2011;25(4):411-419. 